\title{
Plasma BRAF Mutation Detection for the Diagnostic and Monitoring Trajectory of Patients with LDH-High Stage IV Melanoma
}

\author{
Sofie H. Tolmeijer ${ }^{1}$ (D), Rutger H. T. Koornstra ${ }^{2,3}$, Jan Willem B. de Groot ${ }^{4}$, Maartje J. Geerlings ${ }^{5}$, \\ Dirk H. van Rens ${ }^{2}$, Marye J. Boers-Sonderen ${ }^{2}$, Jack A. Schalken ${ }^{6}$, Winald R. Gerritsen ${ }^{2}$, \\ Marjolijn J. L. Ligtenberg ${ }^{5,7}$ (D) and Niven Mehra ${ }^{2, *(D)}$
}

\section{check for} updates

Citation: Tolmeijer, S.H.; Koornstra, R.H.T.; de Groot, J.W.B.; Geerlings,

M.J.; van Rens, D.H.; Boers-Sonderen, M.J.; Schalken, J.A.; Gerritsen, W.R.; Ligtenberg, M.J.L.; Mehra, N. Plasma BRAF Mutation Detection for the Diagnostic and Monitoring Trajectory of Patients with LDH-High Stage IV Melanoma. Cancers 2021, 13, 3913. https://doi.org/10.3390/cancers13153913

Academic Editor: Nicolas Dumaz

Received: 12 July 2021

Accepted: 30 July 2021

Published: 3 August 2021

Publisher's Note: MDPI stays neutral with regard to jurisdictional claims in published maps and institutional affiliations.

Copyright: (c) 2021 by the authors. Licensee MDPI, Basel, Switzerland. This article is an open access article distributed under the terms and conditions of the Creative Commons Attribution (CC BY) license (https:/ / creativecommons.org/licenses/by/ $4.0 /)$.
1 Department of Medical Oncology, Radboud Institute for Molecular Life Sciences, Radboud University Medical Center, 6525 GA Nijmegen, The Netherlands; sofie.tolmeijer@radboudumc.nl

2 Department of Medical Oncology, Radboud Institute for Health Sciences, Radboud University Medical Center, 6525 GA Nijmegen, The Netherlands; RKoornstra@Rijnstate.nl (R.H.T.K.);

Dirk.vanRens@radboudumc.nl (D.H.v.R.); Marye.Boers-Sonderen@radboudumc.nl (M.J.B.-S.);

Winald.Gerritsen@radboudumc.nl (W.R.G.)

3 Department of Medical Oncology, Rijnstate Hospital, 6815 AD Arnhem, The Netherlands

4 Department of Medical Oncology, Isala Oncology Center, 8025 AB Zwolle, The Netherlands; j.w.b.de.groot@isala.nl

5 Department of Human Genetics, Radboud Institute for Molecular Life Sciences, Radboud University Medical Center, 6525 GA Nijmegen, The Netherlands; Maartje.Geerlings@radboudumc.nl (M.J.G.); Marjolijn.Ligtenberg@radboudumc.nl (M.J.L.L.)

6 Department of Urology, Radboud Institute for Molecular Life Sciences, Radboud University Medical Center, 6525 GA Nijmegen, The Netherlands; Jack.Schalken@radboudumc.nl

7 Department of Pathology, Radboud Institute for Molecular Life Sciences, Radboud University Medical Center, 6525 GA Nijmegen, The Netherlands

* Correspondence: Niven.Mehra@radboudumc.nl

Simple Summary: For patients with metastatic melanoma, a rapid BRAF mutation assessment is vital to reveal the treatment options per patient. Additionally, close monitoring of the disease during treatment is essential to allow for adjustments in the treatment strategy when needed. The aim of this prospective study was to confirm the clinical validity of circulating tumor DNA (ctDNA) for minimally invasive $B R A F$ mutation testing and treatment monitoring of metastatic melanoma patients with elevated lactose dehydrogenase serum levels. We observed that ctDNA-based BRAF mutation testing was a reliable and minimally-invasive alternative to tissue-based testing in $98 \%$ of all patients and was $100 \%$ specific. The changes in ctDNA levels during therapy appeared helpful for disease monitoring and outperformed other blood-based biomarkers in predicting treatment response.

Abstract: For patients with newly diagnosed metastatic melanoma, rapid BRAF mutation (mBRAF) assessment is vital to promptly initiate systemic therapy. Additionally, blood-based biomarkers are desired to monitor and predict treatment response. Circulating tumor DNA (ctDNA) has shown great promise for minimally invasive mBRAF assessment and treatment monitoring, but validation studies are needed. This prospective study utilized longitudinal plasma samples at regular timepoints $(0$, $6,12,18$ weeks) to address the clinical validity of ctDNA measurements in stage IV melanoma patients with elevated serum lactate dehydrogenase ( $\mathrm{LDH}>250 \mathrm{U} / \mathrm{L}$ ) starting first-line systemic treatment. Using droplet digital PCR, the plasma mBRAF abundance was assessed in 53 patients with a BRAFV600 tissue mutation. Plasma mBRAF was detected in 50/51 patients at baseline (98\% sensitivity; median fraction abundance of $19.5 \%$ ) and $0 / 17$ controls (100\% specificity). Patients in whom plasma mBRAF became undetectable during the first 12-18 weeks of treatment had a longer progression-free survival (30.2 vs. 4.0 months; $p<0.001)$ and cancer-specific survival (not reached vs. 10.2 months; $p<0.001$ ) compared to patients with detectable mBRAF. The ctDNA dynamics outperformed LDH and S100 dynamics. These results confirm the clinical validity of ctDNA measurements as a minimally invasive biomarker for the diagnostic and monitoring trajectory of patients with LDH-high stage IV melanoma. 
Keywords: circulating tumor DNA (ctDNA), BRAF; biomarker; lactate dehydrogenase (LDH), S100; melanoma

\section{Introduction}

Metastatic melanoma is the most aggressive and lethal form of skin cancer [1-3]. Two therapeutic approaches have become standard of care for this disease: targeted therapy and immunotherapy. Targeted therapy (BRAF/MEK inhibitor) has a rapid anti-tumor effect and is of benefit to the majority of patients. However, this therapy is limited to those harboring a BRAFV600 mutation (mBRAF) in their tumor, and resistance commonly occurs within 12 months [4,5]. Immunotherapy, on the other hand, can achieve long-term disease control and is independent of $B R A F$ status. Still, immunotherapy does not demonstrate sufficient anti-tumor activity in $50-70 \%$ of patients and is associated with a higher incidence of grade 3-4 toxicity [6-9].

To determine the best treatment strategy per patient, it is essential to rapidly assess mBRAF status and closely monitor treatment response. In current practice, mBRAF status is determined from routinely performed tumor biopsies, but this remains an invasive and time-consuming method. Treatment response is monitored by radiographic imaging, which limits frequent measurements and has difficulties distinguishing pseudo-progression from true progression, particularly following checkpoint inhibitors [10,11]. Therefore, alternative strategies have been investigated to improve current practice, including the use of circulating tumor DNA (ctDNA). ctDNA is released into the bloodstream by apoptosis and necrosis of tumor cells [12], enabling the detection of mBRAF from blood. In addition, easily obtainable repetitive blood draws allow close monitoring of ctDNA dynamics in relation to treatment response.

Previous studies established ctDNA analysis as a highly specific tool for mBRAF detection, but ctDNA-based mBRAF detection can vary in sensitivity (56-90\%) [13-19]. The varying sensitivity can be explained by the ctDNA quantity in patients, which is in turn dependent on the tumor burden and location of the tumor. For instance, in patients with M1c disease, it appeared 2-5 times more likely to detect ctDNA compared to M1a/b disease [20-22]. In addition, elevated lactate dehydrogenase (LDH), associated with tumor cells outgrowing their blood supply, is associated with 30-50 times higher ctDNA levels $[14,23,24]$. Consequently, the sensitivity of ctDNA-based mBRAF assessment can vary per patient, hampering the implementation of ctDNA-based mBRAF assessment in routine patient care.

Besides the diagnostic application of ctDNA for mBRAF assessment, the ctDNA burden is prognostic for patient outcome. Similar to LDH, the amount of ctDNA at the start of treatment appears prognostic for the progression-free survival (PFS) and overall survival (OS) of patients with metastatic melanoma $[16,17,21,25,26]$. Additionally, changes in ctDNA were shown to be relevant for the monitoring of treatment response. For example, a conversion of ctDNA from detectable to undetectable levels during immunotherapy or targeted therapy was shown to reflect a 3-7 times longer PFS and 4-8 times longer OS [16,18,21,27]. Small and retrospective studies indicate that ctDNA outperforms the other blood-based biomarkers for melanoma, LDH and S100, in predicting patient outcome [15,28,29].

To validate the current applications for ctDNA, prospective clinical validation studies are needed using blood samples at regular time points and standardized blinded assessment of outcome parameters. Syeda and colleagues published the first large clinical validation study showing the potential of ctDNA as an independent biomarker for targeted therapy in patients with advanced melanoma [26]. Plasma mBRAF was detected in 93\% (320/345) of all patients using droplet digital PCR (ddPCR) [26]. In patients with elevated LDH levels, the sensitivity was $98 \%$, showing a great promise for ctDNA-based mBRAF assessment, particularly in LDH-high stage IV melanoma patients. As elevated LDH is associated with a 50\% shorter OS compared to patients with normal LDH [30], prompt 
initiation of treatment and close treatment monitoring is essential for these patients. Interestingly, Syeda and colleagues observed a better predictive value of ctDNA dynamics for the PFS and OS in LDH-high patients compared to LDH-normal patients [26]. Unfortunately, longitudinal sampling beyond 4 weeks was missing in this study, and the ctDNA dynamics were not compared to other blood-based biomarkers.

The current study aimed to confirm and expand on the clinical validity of ctDNA measurements for diagnostic and monitoring trajectory of patients with LDH-high metastatic melanoma starting their first-line of systemic treatment. Utilizing longitudinal and prospectively collected plasma samples at fixed timepoints up to 18 weeks of treatment, accompanied by radiographic imaging and evaluation of other blood-based biomarkers, we aim to elaborate on the potential of ctDNA measurements for systemic treatment monitoring in LDH-high stage IV melanoma patients.

\section{Materials and Methods}

\subsection{Patient Cohort and Study Design}

Patients with stage IV metastatic melanoma were enrolled in the study between March 2017 and June 2020. All patients had a confirmed BRAFV600 mutation (mBRAF) in tissue based on routine diagnostic tests. All patients had elevated serum LDH ( $>250 \mathrm{U} / \mathrm{L})$ and were naïve for both immune checkpoint blockade agents and BRAF/MEK inhibitors. Written consent was obtained from all patients as approved by the local medical ethical committee (dossier number 2016-2769, December 2016). Patients underwent baseline characterization, including physical examination, blood marker evaluation, and radiographic tumor assessment. Patients started with either BRAF/MEK inhibitors or immune checkpoint blockade upon inclusion and underwent clinical evaluation every 6 weeks, which included blood collection and CT scans. CT results were assessed by RECIST 1.1 criteria [31], which distinguishes between complete response (CR), partial response (PR), stable disease (SD), or progressive disease (PD).

In order to confirm the specificity of ctDNA-based mBRAF detection, blood was also collected from three LDH-high melanoma patients without mBRAF in their tumor and 14 healthy controls.

\subsection{Cell-Free DNA Isolation and ctDNA Quantification}

Blood was collected at baseline and after 6, 12, and 18 weeks of treatment using EDTA tubes. Within 4 hours, the blood samples were first centrifuged at $120 \times \mathrm{g}$ for $20 \mathrm{~min}$ to separate plasma from blood cells. Afterward, plasma was centrifuged at $360 \times g$ for $20 \mathrm{~min}$ to remove platelets. Finally, the plasma was centrifuged at $14,000 \times g$ for $10 \mathrm{~min}$ to remove cellular debris. Plasma was stored at $-80^{\circ} \mathrm{C}$ until further processing.

Total cell-free circulating DNA was extracted from approximately $2 \mathrm{~mL}$ of plasma using the QIAamp Circulating Nucleic Acid Kit (Qiagen) according to the manufacturer's protocol and eluted in $30 \mu \mathrm{L}$ low-TE buffer. The DNA concentration was quantified using Qubit (ThermoFisher), and the quality was checked on a Fragment Analyzer (Agilent high sensitivity genomic DNA kit \#DNF-488-0500). Next, the presence of mBRAF ctDNA copies was assessed with the droplet digital PCR (ddPCR) BRAFV600 screening kit (\#12001037, BioRad), which can detect BRAF p.V600E (c.1799T>A), p.V600R (c.1798_1799delinsAG), and p.V600K (c.1798_1799delinsAA) mutations. All samples were measured in duplicate. A binominal distribution was used to calculate the theoretical sensitivity of detecting mBRAF per sample based on the available input material (Figure S1). Samples with two or more mutant droplets were considered ctDNA positive.

To convert cell-free DNA concentration-units from ng per $\mathrm{mL}$ plasma to copies per $\mathrm{mL}$ plasma, we multiplied the concentrations by a factor of 303, assuming that the mass of a haploid genome is $3.3 \mathrm{pg}$. Subsequently, the ctDNA copies per mL plasma could be calculated based on the fractional abundance of mBRAF and the total cell-free DNA copies per mL plasma. 


\subsection{Statistical Analysis}

The correlation between continuous variables was calculated using Spearman rank correlation statistics. Differences in ctDNA levels concerning the absence or presence of specific metastasis sites were calculated using an unpaired two samples Wilcoxon test. Time-to-event outcomes, including PFS and melanoma cancer-specific survival (CSS), were described via the Kaplan-Meier method. PFS and CSS were defined as the time from the start of therapy to the date of first reported PD for PFS and death as a consequence of melanoma for CSS. PFS and CSS curves were stratified according to patient characteristics and clinicopathologic features and compared using Cox-regression models. For the Cox-regression models, the baseline ctDNA copies were log-transformed for a normal distribution and assessed as a continuous variable. For assessment of ctDNA dynamics in longitudinal samples, ctDNA results were dichotomized as detectable (positive) or undetectable (negative) after 12-18 weeks of treatment. S100 and LDH dynamics were also dichotomized as below the upper limit of normal or above the upper limit of normal after 12-18 weeks. Due to missing data and a limited number of events for CSS $(n=16)$, a multivariable Cox-regression was only used to evaluate PFS. This multivariate Cox-regression analysis included all variables significantly associated with PFS in the univariate analysis $(p<0.05)$.

\section{Results}

\subsection{Patient Characteristics}

A total of 53 patients with LDH-high metastatic melanoma were included in this study (Table 1). Half of these patients were treated with combination immunotherapy (ipilimumab + nivolumab), while the other half was first treated with combination BRAF/MEK inhibitors before starting with immunotherapy. As this translational work is part of an ongoing clinical trial, we cannot disclose patient treatment specifics. The median follow-up duration was 12.3 months (range 0-38.1 months). Fifty-eight percent of patients were alive at the time of analysis, and $42 \%$ had an ongoing treatment response.

Table 1. Baseline patient characteristics.

\begin{tabular}{lc}
\hline \multicolumn{1}{c}{ Total Patients, $\boldsymbol{n}(\mathbf{\%})$} & $\mathbf{5 3} \mathbf{( 1 0 0 \% )}$ \\
\hline Sex, $n(\%)$ & $19(36 \%)$ \\
Female & $34(64 \%)$ \\
Male & $61(28-78)$ \\
Age & \\
Median years (range) & $34(64 \%)$ \\
ECOG, $n$ (\%) & $16(30 \%)$ \\
0 & $3(6 \%)$ \\
1 & \\
2 & $28(53 \%)$ \\
Initiated treatment, $n(\%)$ & $25(47 \%)$ \\
Immunotherapy & $357(261-1560)$ \\
BRAF/MEK inhibitor & \\
LDH (U/L) & $1.43(0.06-10.97)$ \\
Median (range) & \\
S100 (ng/mL) & $9(17 \%)$ \\
Median (range) & $34(64 \%)$ \\
Metastasis location, $n(\%)$ & $23(43 \%)$ \\
Skin & $21(40 \%)$ \\
Lymph node & $19(36 \%)$ \\
Lung & $12.3(0-38.1)$ \\
Kidney & \\
Liver & \\
Follow-up & \\
Median months (range) & \\
\hline
\end{tabular}




\section{2. ctDNA-Based mBRAF Assessment}

In total, 153 blood samples were collected for ctDNA analysis (data available in Table S1). This included a baseline sample for 51/53 (96\%) patients and longitudinal follow-up for 40/53 (75\%) patients. Of all baseline plasma samples, mBRAF was detected in 50/51 (98\%) with a median fractional abundance of $19.5 \%$ (range $0.2-66.5 \%$ ). The one patient for whom mBRAF could not be detected had M1b disease and the smallest cumulative RECIST target lesions of the cohort.

Figure $1 \mathrm{~A}$ visualizes the baseline plasma mBRAF abundance in relation to other baseline characteristics, such as LDH levels and metastasis sites. mBRAF abundance moderately correlated with levels of LDH (Figure 1B, $\rho=0.50, p<0.001$ ), weakly correlated with S100 levels (Figure 1C, $\rho=0.35, p=0.03$ ), and strongly correlated with total cell-free circulating DNA (Figure 1D, $\rho=0.83, p<0.001$ ). No association was found between the mBRAF levels and the RECIST sum of the target lesion diameters (SLD) $(p=0.74)$, but higher mBRAF levels were observed in patients with liver metastasis (Figure 1E, $p=0.05$ ).

A

A $\quad$\begin{tabular}{lllllllllllllllll} 
Patient & \\
\hline
\end{tabular}

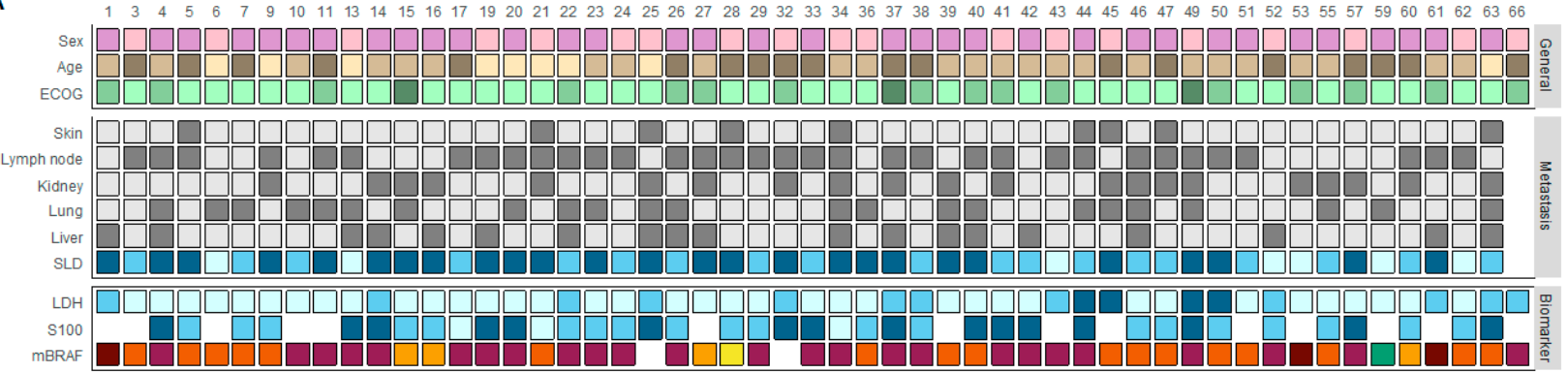

$\begin{array}{llllllll}\text { Sex } & \text { Age [years] } & \text { ECOG } & \text { Metastasis present } & \text { SLD [mm] } & \text { LDH [U/L] } & \text { S100 [ng/mL] } & \text { mBRAF [copies/mL plasma] } \\ \square \text { Male } & \square<50 & \square & \square \text { No } & \square<50 & \square 250-500 & \square<0.2 & \square \text { Undetectable } \\ \square \text { Female } & \square>65 & \square 1 & \square \text { Yes } & \square 50-100 & \square 500-750 & \square>2.0 & \square 1-10 \\ & & \square 2 & & \square>100 & \square>750 & \square-1000\end{array}$
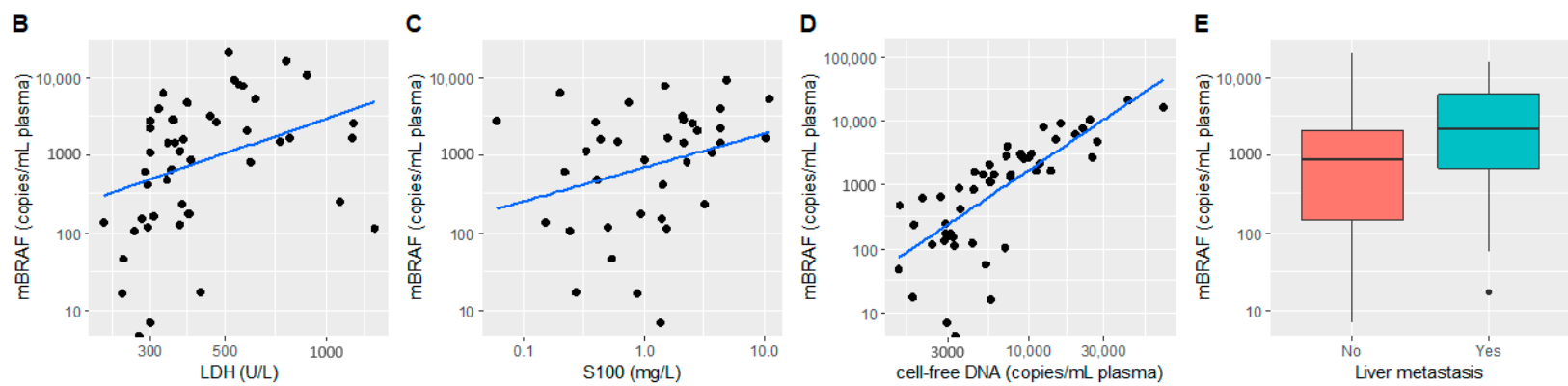

Figure 1. Baseline patient characteristics and clinicopathologic features in relation to baseline plasma mBRAF copies. (A) Schematic overview illustrating the patient characteristics (sex, age, ECOG), metastasis sites and blood-based biomarkers (LDH, S100) relative to the plasma mBRAF copies; (B) Correlation between plasma mBRAF copies and LDH levels ( $\rho=0.50$, $p<0.001)$; (C) Correlation between plasma mBRAF copies and S100 levels $(\rho=0.35, p=0.03)$; (D) Correlation between plasma mBRAF copies and total cell free circulating DNA copies $(\rho=0.83, p<0.001)$; (E) Association between plasma mBRAF copies and the presence of liver metastasis $(p=0.05)$.

To determine the specificity of ctDNA-based mBRAF detection, plasma of 17 controls was tested for the presence of mBRAF. Fourteen of these controls were healthy individuals, and three were patients with LDH-high metastatic melanoma but without mBRAF in their tumor. All the plasma samples tested negative for mBRAF (Table S1). Combined, this indicates that ctDNA-based mBRAF detection has a specificity of $100 \%$ and sensitivity of $98 \%$ in LDH-high stage IV melanoma patients. 


\section{3. ctDNA Dynamics and Treatment Response}

After treatment initiation, we investigated ctDNA dynamics in relation to the treatment response. For 40 patients, follow-up plasma samples were available up to 12-18 weeks and/or at progression. An overview of the longitudinal blood-biomarker assessments available per patient is given in Table S3. The ctDNA dynamics of these patients could be divided into two groups: (1) 23 patients in whom plasma mBRAF became undetectable during the first 12-18 weeks of treatment, including the patient who was tested mBRAFnegative at baseline, and (2) 17 patients in whom plasma mBRAF remained detectable (or became detectable again) during the first 12-18 weeks of treatment. Figure 2 visualizes the ctDNA dynamics in both groups, referred to as (1) ctDNA negative and (2) ctDNA positive. Figure S2 includes the results on the S100 and LDH dynamics in the ctDNA dynamics groups.

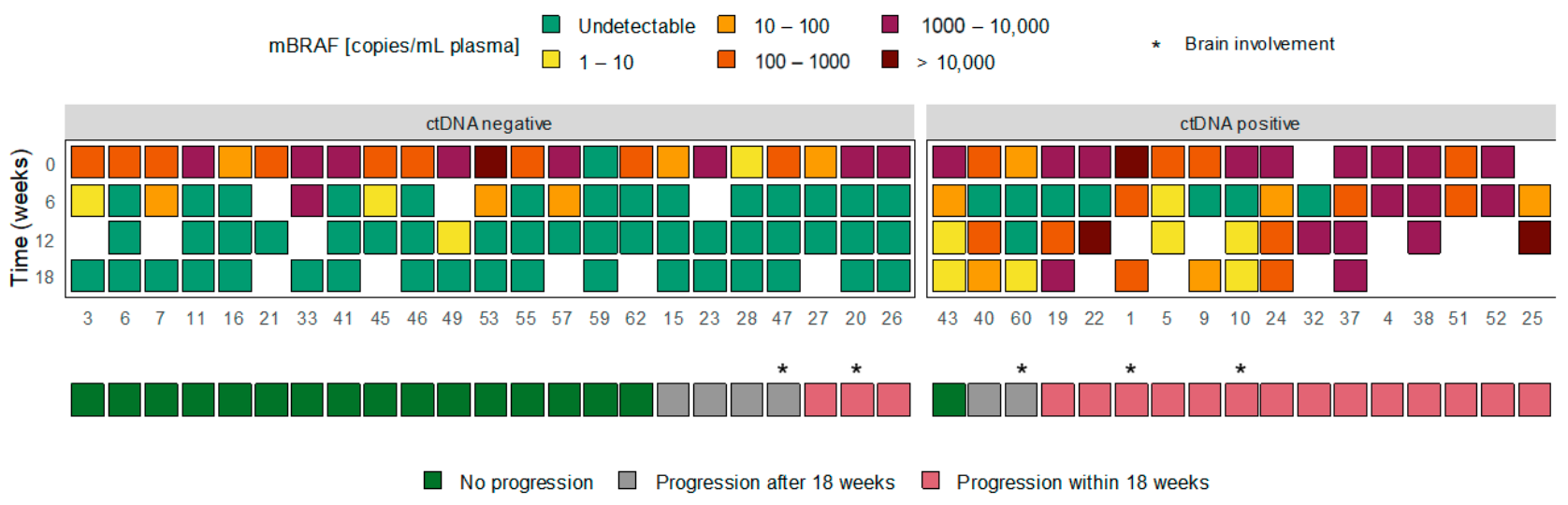

Figure 2. Overview of the ctDNA dynamics (mBRAF copies/mL plasma) related to treatment response within 18 weeks. Patients were stratified according to their ctDNA levels at 12-18 weeks, being either detectable (ctDNA positive) or undetectable (ctDNA negative).

In the ctDNA negative group, only three $(13 \%)$ patients experienced disease progression within 18 weeks. All three patients had an ongoing response per RECIST1.1 of their target lesions but developed one or more new lesions. One of the three patients developed only one new lesion that was located in the brain. Four $(17 \%)$ patients in the ctDNA negative group had disease progression after 18 weeks, and 16 (70\%) had an ongoing treatment response at the time of analysis. In the ctDNA positive group, $14(82 \%)$ patients developed disease progression within 18 weeks and $2(12 \%)$ after 18 weeks. Only one $(6 \%)$ patient had a continuing treatment response. The ctDNA content of this patient was still declining from baseline to the last measured timepoint.

\section{4. ctDNA Dynamics Associates with the PFS and CSS}

Next, we investigated the ctDNA dynamics in relation to PFS and CSS. Figure 3A demonstrates that patients with undetectable ctDNA after 12-18 weeks of treatment had a 7.4 times longer median PFS compared to patients with still detectable ctDNA (30.2 vs. 4.0 months; hazard ratio (HR) 12.6 (95\% confidence interval $[95 \% \mathrm{CI}] 4.3-36.8)$ ). A similar difference was observed for CSS (Figure 3B; not reached vs. 10.2 months; HR 14.6 (95\% CI 3.3-64.6)).

Other parameters that were significantly associated with a shorter PFS in the univariable analysis included the presence of liver metastasis, the amount of mBRAF copies at baseline, and the S100 dynamics (being above or under the upper limit of normal after 12-18 weeks) (Table 2). Interestingly, only ctDNA dynamics remained significant in a multivariable model (Table 2). The univariable analysis for CSS revealed that similar variables associated with a shorter PFS were also associated with a shorter CSS (Table S2). Due to limited events in the CSS analysis and missing data (Table S3), we did not perform a multivariable analysis with all significantly associated variables for CSS. Still, ctDNA 
dynamics was the strongest prognostic variable in the univariate analysis for both PFS and CSS.

A

+ ctDNA negative + ctDNA positive

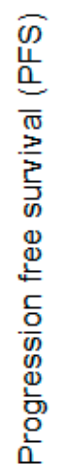

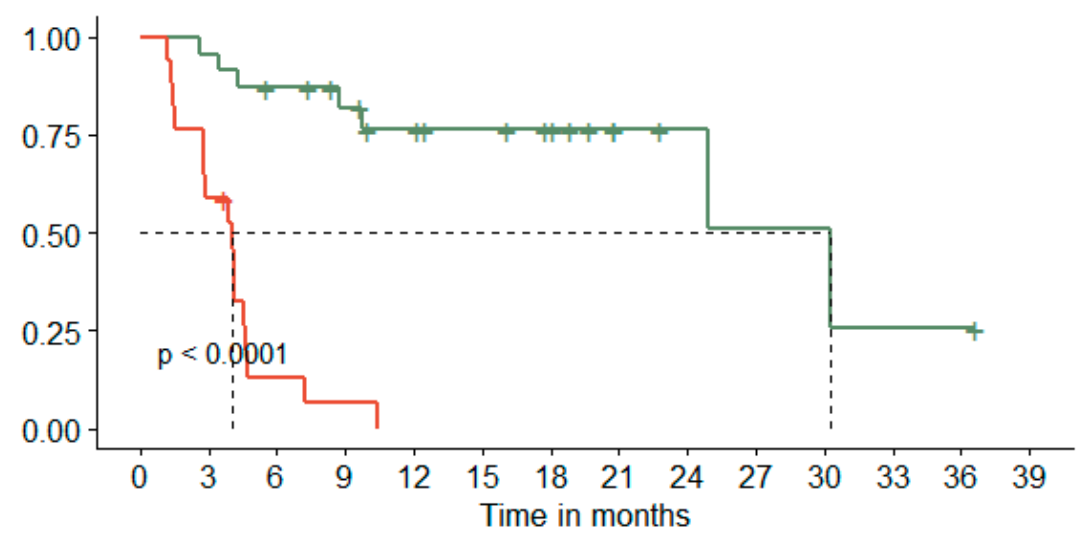

Number at risk

\begin{tabular}{|c|c|c|c|c|c|c|c|c|c|c|c|c|c|c|}
\hline $\begin{array}{l}\text { ctDNA negative } \\
\text { ctDNA positive }\end{array}$ & $\begin{array}{l}23 \\
17 \\
\end{array}$ & $\begin{array}{l}22 \\
10 \\
\end{array}$ & $\begin{array}{c}19 \\
2\end{array}$ & $\begin{array}{c}16 \\
1\end{array}$ & $\begin{array}{c}13 \\
0\end{array}$ & $\begin{array}{c}11 \\
0\end{array}$ & $\begin{array}{l}9 \\
0 \\
\end{array}$ & $\begin{array}{l}4 \\
0 \\
\end{array}$ & $\begin{array}{l}3 \\
0 \\
\end{array}$ & $\begin{array}{l}2 \\
0 \\
\end{array}$ & $\begin{array}{l}2 \\
0 \\
\end{array}$ & $\begin{array}{l}1 \\
0\end{array}$ & $\begin{array}{l}1 \\
0\end{array}$ & $\begin{array}{l}0 \\
0\end{array}$ \\
\hline & 0 & 3 & 6 & 9 & 12 & 15 & 18 & 21 & 24 & 27 & 30 & 33 & 36 & 39 \\
\hline
\end{tabular}

$\mathbf{B}$

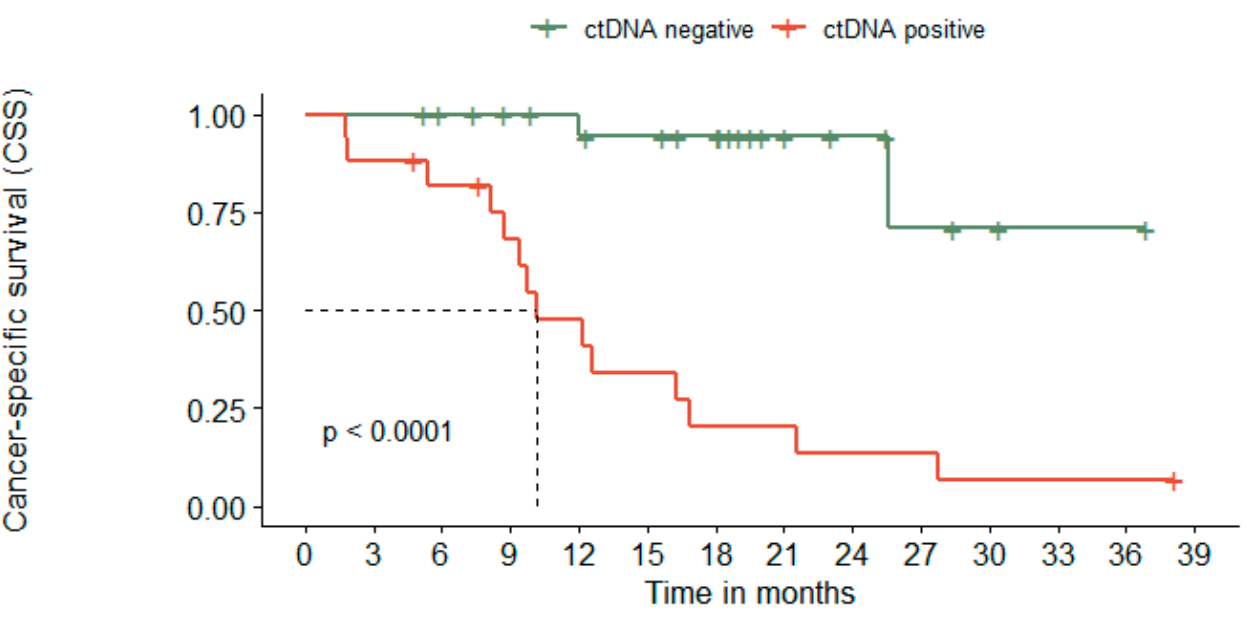

Number at risk

\begin{tabular}{|c|c|c|c|c|c|c|c|c|c|c|c|c|c|c|}
\hline $\begin{array}{l}\text { ctDNA negative - } \\
\text { ctDNA positive - }\end{array}$ & $\begin{array}{l}23 \\
17 \\
\end{array}$ & $\begin{array}{l}23 \\
15 \\
\end{array}$ & $\begin{array}{l}21 \\
13 \\
\end{array}$ & $\begin{array}{l}19 \\
10 \\
\end{array}$ & $\begin{array}{l}17 \\
7 \\
\end{array}$ & $\begin{array}{l}16 \\
5 \\
\end{array}$ & $\begin{array}{c}14 \\
3 \\
\end{array}$ & $\begin{array}{l}8 \\
3 \\
\end{array}$ & $\begin{array}{l}5 \\
2 \\
\end{array}$ & $\begin{array}{l}3 \\
2 \\
\end{array}$ & $\begin{array}{l}2 \\
1 \\
\end{array}$ & $\begin{array}{l}1 \\
1 \\
\end{array}$ & $\begin{array}{l}1 \\
1 \\
\end{array}$ & $\begin{array}{l}0 \\
0 \\
\end{array}$ \\
\hline & 0 & 3 & 6 & 9 & 12 & 15 & 18 & 21 & 24 & 27 & 30 & 33 & 36 & 39 \\
\hline
\end{tabular}

Figure 3. ctDNA dynamics related to (A) progression-free survival (PFS) and (B) cancer-specific survival (CSS). Patients were stratified according to their ctDNA levels at 12-18 weeks, being either detectable (ctDNA positive) or undetectable (ctDNA negative). 
Table 2. Factors associated with the progression-free survival (PFS). HR = hazard ratio, 95\% CI = 95\% confidence interval, $\mathrm{ULN}=$ upper limit of normal.

\begin{tabular}{|c|c|c|c|c|c|c|c|}
\hline \multirow[b]{3}{*}{ Variable } & & \multicolumn{6}{|c|}{ Progression-Free Survival } \\
\hline & & \multicolumn{3}{|c|}{ Univariate Analysis } & \multicolumn{3}{|c|}{ Multivariate Analysis } \\
\hline & & HR & $95 \%$ CI & $p$-Value & HR & $95 \%$ CI & $p$-Value \\
\hline Liver metastasis & Present vs. absent & 3.65 & $1.73-7.71$ & $<0.001$ & 1.38 & $0.36-5.3$ & 0.643 \\
\hline ctDNA baseline & $\log 10$ (mBRAF per mL plasma) & 1.68 & $1.07-2.64$ & 0.02 & 1.26 & $0.67-2.4$ & 0.473 \\
\hline S100 dynamics & Above vs. below ULN at $12-18$ weeks & 5.5 & $2.21-13.71$ & $<0.001$ & 0.95 & $0.23-4.0$ & 0.94 \\
\hline ctDNA dynamics & Positive vs. negative at $12-18$ weeks & 12.57 & $4.30-36.76$ & $<0.001$ & 18.75 & $3.55-98.9$ & $<0.001$ \\
\hline
\end{tabular}

\section{Discussion}

The current study confirmed that ctDNA could be a valuable diagnostic and predictive tool in patients with LDH-high metastatic melanoma. Before treatment initiation, ctDNAbased mBRAF assessment was shown to be highly sensitive and specific for these patients. Using prospectively collected longitudinal data at fixed timepoints, it was shown that ctDNA dynamics can be used to monitor treatment response. The ctDNA dynamics defined favorable and unfavorable profiles that could be used as an independent predictor of longterm response and survival and may eventually be used to guide treatment adaptations.

To determine optimal therapeutic choices in metastatic melanoma, knowledge on the $B R A F$ status is vital. Particularly in newly diagnosed, symptomatic, LDH-high metastatic melanoma, prompt treatment initiation with BRAF/MEK inhibition allows for a rapid tumor and clinical response in patients harboring mBRAF. At present, BRAF status is assessed using a tumor biopsy, but ctDNA-based mBRAF detection could become a new standard being a less-invasive and faster strategy for accurate $B R A F$ assessment (within days instead of weeks). With the level of ctDNA in blood to a large extent dependent on tumor burden $[4,20,21]$, we hypothesized that ctDNA-based mBRAF detection would be most reliable in patients with LDH-high metastatic disease. Elevated LDH is associated with tumors outgrowing their blood supply [32] and has clinical utility in melanoma. LDH is used as a classifier for the American joint committee on cancer staging [33] and is a strong prognostic biomarker for metastatic melanoma independent of treatment $[34,35]$. Moreover, previous studies revealed a clear correlation between LDH and ctDNA levels $(\mathrm{R}=0.50-0.76)[14-16,23]$.

A moderate correlation between LDH and ctDNA was still observed in this study even though patients were preselected for elevated LDH levels $(\rho=0.50)$. More importantly, $50 / 51$ patients positive for mBRAF by tumor tissue test also had detectable mBRAF in plasma resulting in a $98 \%$ sensitivity of the ctDNA-based assay. This is similar to the results of Syeda and colleagues, who also observed a 98\% sensitivity among 125 patients with LDHhigh advanced melanoma [26]. The sensitivity is higher compared to previous studies in which patients were not treatment-naïve or preselected for elevated LDH (56-90\%) [13-19]. The sensitivity was independent of the assay threshold that was previously reported to affect sensitivity [18]. The specificity obtained in this study (100\%) was comparable to other studies [13-19,36]. As approximately $40 \%$ of all metastatic melanoma patients have elevated serum LDH [4], ctDNA-based mBRAF detection can become a reliable alternative to tissue-based testing for a substantial number of patients to guide the initial choice of systemic therapy. The next step for the implementation of ctDNA-based mBRAF testing in the clinic would be to offer ctDNA-based mBRAF testing in parallel to tissue testing and compare the turn-around time, sensitivity, and costs.

Besides the baseline ctDNA detection, the ctDNA changes in relation to treatment response were investigated in this study. Figure 2 illustrates that the majority of patients in whom ctDNA became undetectable during the first 12-18 weeks (ctDNA negative) had a favorable treatment response. Disease progression within 18 weeks was only observed in three patients. Interestingly, all these patients had responding RECIST target lesions but developed one or more new lesions. In patient 20, the new lesion was located in the brain, 
potentially explaining the absence of ctDNA in plasma [18,21,37]. Patient 26 was later diagnosed with myelofibrosis, which might explain the new lesions on the CT-scan without mBRAF detection in blood. This patient was switched to BRAF/MEK inhibitors after the new lesions were observed and had a complete response following the next 2.5 years, which is a remarkable duration of response to BRAF/MEK inhibitors. In contrast to the ctDNA negative group, most patients in the ctDNA positive group experienced disease progression within the first 18 weeks of treatment. Only one patient had a long-term treatment response beyond 18 weeks. This patient showed an ongoing decline in ctDNA copies from baseline in all measured timepoints and a longer ctDNA evaluation period may therefore have resulted in undetectable mBRAF level following the 18-week period. Altogether, the data suggest that longitudinal measurements of ctDNA during treatment could help monitor treatment response.

When translating the observations from Figure 2 to a survival analysis, a clear association was observed between ctDNA dynamics and time to progression or death. Patients with undetectable levels of ctDNA after 12-18 weeks of treatment had a 7.6 time longer median PFS compared to patients with detectable ctDNA (Figure 3A). Moreover, only two melanoma-related deaths were observed in this group following a median follow-up of 18.4 months and included the death of patient 20, who developed brain metastasis (Figure 3B). These observations are in line with previous literature, describing comparable hazard ratios for the ctDNA detectability at 3 to 12 weeks $[18,21,27]$. Similarly, around $50 \%$ of the patients with detectable ctDNA at the start of treatment convert to undetectable ctDNA after a few weeks of treatment $[18,27]$.

Importantly, ctDNA dynamics improved discrimination between progressing and non-progressing patients within the observation period of 18 weeks when compared to S100 and LDH dynamics (Figure S2; Table S2; Table 2). This is in line with results from other small studies and retrospective studies $[15,28,29]$. Overall, the ctDNA dynamics resembled S100 dynamics despite a weak correlation between the variables at baseline. Nonetheless, S100 dynamics misclassified five patients that were correctly classified by ctDNA dynamics (Figure S2). LDH dynamics misclassified nine patients and was not significantly associated with PFS in the univariate analysis (Figure S2; Table S2). Only ctDNA dynamics remained significant in a multivariate Cox-regression model for PFS after correction for the significant baseline characteristics and S100 dynamics (Table 2). Therefore, ctDNA dynamics seems a very specific and valuable biomarker to distinguish long-term responders from patients who may benefit from an early switch to an alternative treatment strategy or possibly treatment intensification. Ongoing randomized trials are evaluating whether ctDNA-informed early treatment switch may improve outcome compared to standard of care; this includes a randomized phase II trial in patients with metastatic melanoma treated with dabrafenib and trametinib, where following a predefined ctDNA drop of $\geq 80 \%$, patients are switched to nivolumab and ipilimumab (NCT03808441). In the adjuvant setting, a phase 3 randomized, blinded trial will investigate whether ctDNAinformed early treatment initiation with nivolumab has a superior outcome to the standard of care in patients following resection of stage IIB/C melanoma (NCT04901988).

To conclude, this study on prospectively collected material underlines the potential of ctDNA assessment as a diagnostic and predictive tool for patients with LDH-high metastatic melanoma. Limitations of this study included the small number of patients and a ctDNA follow-up that stopped at 18 weeks. Future studies will be needed to investigate the clinical utility of ctDNA-based mBRAF assessment in routine practice and help identify optimal use of longitudinal ctDNA follow-up. Nevertheless, this study supports the next steps in the implementation of ctDNA assessments in routine clinical care of metastatic melanoma patients.

\section{Conclusions}

In conclusion, the current study confirmed the clinical validity of ctDNA-based mBRAF detection as an alternative to tissue-based testing for patients with LDH-high 
metastatic melanoma. Using prospectively collected blood samples at regular timepoint, the study underlines the potential of ctDNA dynamics to monitor and independently predict treatment response.

Supplementary Materials: The following are available online at https:/ /www.mdpi.com/article/10 .3390 / cancers13153913/s1: Figure S1: The theoretical sensitivity of the droplet digital PCR (ddPCR). In the graph the theoretical minimally detectable fractional abundance of mBRAF is visualized against the input DNA. The calculation is based on the binominal distribution with the assumption that a minimal of 2 mutant droplets should be detected by the ddPCR. Figure S2: The ctDNA dynamics in relation to the S100 and LDH dynamics. (a) Overview of the ctDNA dynamics (mBRAF copies/mL plasma) related to treatment response within 18 weeks. Patients were stratified according to their ctDNA levels at 12-18 weeks, being either detectable (ctDNA positive) or undetectable (ctDNA negative). (b) The S100 and LDH dynamics of the patients visualized in Figure S2A. Patients in whom S100/LDH levels normalized within 12-18 weeks were marked by a light-blue box. If S100 or LDH levels remained elevated, patients were marked with a dark-blue box. Table S1: All the droplet digital PCR data on the mBRAF assessment in all patient and control samples. Control samples included 14 plasma samples of healthy individuals and three of LDH-high melanoma patients without mBRAF in their tumor. Table S2: Univariable Cox-regression analysis for the progression free survival and cancer-specific survival. $\mathrm{HR}=$ hazard ratio, $95 \% \mathrm{CI}=95 \%$ confidence interval. Table S3: An overview of the blood-based biomarkers assessed per patient at baseline and during treatment.

Author Contributions: Conceptualization, S.H.T., R.H.T.K., M.J.G., W.R.G., M.J.L.L. and N.M.; Methodology, M.J.G., S.H.T. and M.J.L.L.; Software, NA; Validation, R.H.T.K., M.J.G., W.R.G., M.J.L.L. and N.M.; Formal Analysis, S.H.T., M.J.G. and N.M.; Investigation, S.H.T.; Resources, R.H.T.K., J.W.B.d.G., D.H.v.R., M.J.B.-S., J.A.S., W.R.G., M.J.L.L. and N.M.; Data Curation, S.H.T., R.H.T.K.; Writing一Original Draft Preparation, S.H.T.; Writing—Review \& Editing, S.H.T., R.H.T.K., J.W.B.d.G., M.J.G., D.H.v.R., M.J.B.-S., J.A.S., W.R.G., M.J.L.L. and N.M.; Visualization, S.H.T.; Supervision, R.H.T.K., M.J.G., J.A.S., M.J.L.L., W.R.G. and N.M.; Project Administration, N.M.; Funding Acquisition, W.R.G. and N.M. All authors have read and agreed to the published version of the manuscript.

Funding: This research was funded by the Paul Speth Fund.

Institutional Review Board Statement: The study was conducted according to the guidelines of the Declaration of Helsinki and approved by the medical ethics committee region Arnhem-Nijmegen (dossier number 2016-2769, December 2016).

Informed Consent Statement: Informed consent was obtained from all subjects involved in the study.

Data Availability Statement: The data presented in this study are available in Supplementary Table S1.

Acknowledgments: The authors would like to thank Marion van der Putten and Elze VerbeekCamps for the processing of all plasma samples. Additionally, the authors thank Franchette van den Berkmortel for the helpful clinical discussions.

Conflicts of Interest: Rutger Koornstra reports receiving speaker fees from BMS, MSD, and Roche; he takes part in the advisory boards of BMS, MSD, Novartis, Roche, and received research funding from Roche and BMS. Jan Willem de Groot takes part in the advisory boards of Bristol-Myers Squibb, Roch, Pierre Fabre, Servier, MSD, and Novartis. Marye Boers-Sonderen takes part in the advisory boards of Pierre-Fabre, Merck, and Pfizer. Winald Gerritsen reports receiving research funding from Astellas, Amgen, Bayer, Janssen-Cilaq, and Sanofi; speaker fees from Astellas Bayer, Merck Sharp \& Dohme (MSD), and the European Society for Medical Oncology, and he takes part in the advisory boards of Amgen, Bayer, Bristol-Myers Squibb, Curevac, Dendreon, IQVIA, Janssen-Cilag, MSD, Morphosys, and Sanofi. Marjolijn Ligtenberg reports receiving diagnostic innovation grants from AstraZeneca and Bristol-Myers Squibb; speaker fees from Roche, Merck Sharp \& Dohme, and AstraZeneca; and she takes part in the advisory boards of Lilly, Janssen Pharmaceuticals, Merck Sharp \& Dohme, AstraZeneca, Bristol-Myers Squibb, Bayer, and Novartis. Niven Mehra reports receiving research funding from Astellas, Janssen, Pfizer, Roche, and Sanofi; travel support of Astellas and MSD, and takes part in the advisory boards of Roche, MSD, BMS, Bayer, Astellas, and Janssen. 


\section{References}

1. Rigel, D.S.; Carucci, J.A. Malignant melanoma: Prevention, early detection, and treatment in the 21st century. CA Cancer J. Clin. 2000, 50, 215-236. [CrossRef] [PubMed]

2. Guy, G.P., Jr.; Thomas, C.C.; Thompson, T.; Watson, M.; Massetti, G.M.; Richardson, L.C. Vital signs: Melanoma incidence and mortality trends and projections-United States, 1982-2030. MMWR Morb. Mortal Wkly. Rep. 2015, 64, 591-596. [PubMed]

3. Siegel, R.L.; Miller, K.D.; Jemal, A. Cancer statistics, 2019. CA Cancer J. Clin. 2019, 69, 7-34. [CrossRef] [PubMed]

4. Long, G.V.; Flaherty, K.T.; Stroyakovskiy, D.; Gogas, H.; Levchenko, E.; de Braud, F.; Larkin, J.; Garbe, C.; Jouary, T.; Hauschild, A.; et al. Dabrafenib plus trametinib versus dabrafenib monotherapy in patients with metastatic BRAF V600E/K-mutant melanoma: Long-term survival and safety analysis of a phase 3 study. Ann. Oncol. 2017, 28, 1631-1639. [CrossRef]

5. Larkin, J.; Ascierto, P.A.; Dreno, B.; Atkinson, V.; Liszkay, G.; Maio, M.; Mandala, M.; Demidov, L.; Stroyakovskiy, D.; Thomas, L.; et al. Combined vemurafenib and cobimetinib in BRAF-mutated melanoma. N. Engl. J. Med. 2014, 371, 1867-1876. [CrossRef] [PubMed]

6. Topalian, S.L.; Sznol, M.; McDermott, D.F.; Kluger, H.M.; Carvajal, R.D.; Sharfman, W.H.; Brahmer, J.R.; Lawrence, D.P.; Atkins, M.B.; Powderly, J.D.; et al. Survival, durable tumor remission, and long-term safety in patients with advanced melanoma receiving nivolumab. J. Clin. Oncol. 2014, 32, 1020-1030. [CrossRef]

7. Schadendorf, D.; Hodi, F.S.; Robert, C.; Weber, J.S.; Margolin, K.; Hamid, O.; Patt, D.; Chen, T.T.; Berman, D.M.; Wolchok, J.D. Pooled Analysis of Long-Term Survival Data From Phase II and Phase III Trials of Ipilimumab in Unresectable or Metastatic Melanoma. J. Clin. Oncol. 2015, 33, 1889-1894. [CrossRef]

8. Weber, J.S.; Gibney, G.; Sullivan, R.J.; Sosman, J.A.; Slingluff, C.L., Jr.; Lawrence, D.P.; Logan, T.F.; Schuchter, L.M.; Nair, S.; Fecher, L.; et al. Sequential administration of nivolumab and ipilimumab with a planned switch in patients with advanced melanoma (CheckMate 064): An open-label, randomised, phase 2 trial. Lancet Oncol. 2016, 17, 943-955. [CrossRef]

9. Hamid, O.; Puzanov, I.; Dummer, R.; Schachter, J.; Daud, A.; Schadendorf, D.; Blank, C.; Cranmer, L.D.; Robert, C.; Pavlick, A.C.; et al. Final analysis of a randomised trial comparing pembrolizumab versus investigator-choice chemotherapy for ipilimumab-refractory advanced melanoma. Eur. J. Cancer 2017, 86, 37-45. [CrossRef] [PubMed]

10. Hodi, F.S.; Hwu, W.-J.; Kefford, R.; Weber, J.S.; Daud, A.; Hamid, O.; Patnaik, A.; Ribas, A.; Robert, C.; Gangadhar, T.C.; et al. Evaluation of Immune-Related Response Criteria and RECIST v1.1 in Patients With Advanced Melanoma Treated With Pembrolizumab. J. Clin. Oncol. 2016, 34, 1510-1517. [CrossRef]

11. Seymour, L.; Bogaerts, J.; Perrone, A.; Ford, R.; Schwartz, L.H.; Mandrekar, S.; Lin, N.U.; Litière, S.; Dancey, J.; Chen, A.; et al. iRECIST: Guidelines for response criteria for use in trials testing immunotherapeutics. Lancet. Oncol. 2017, 18, e143-e152. [CrossRef]

12. Stroun, M.; Lyautey, J.; Lederrey, C.; Olson-Sand, A.; Anker, P. About the possible origin and mechanism of circulating DNA: Apoptosis and active DNA release. Clinica. Chimica. Acta 2001, 313, 139-142. [CrossRef]

13. Garlan, F.; Blanchet, B.; Kramkimel, N.; Puszkiel, A.; Golmard, J.L.; Noe, G.; Dupin, N.; Laurent-Puig, P.; Vidal, M.; Taly, V.; et al. Circulating Tumor DNA Measurement by Picoliter Droplet-Based Digital PCR and Vemurafenib Plasma Concentrations in Patients with Advanced BRAF-Mutated Melanoma. Target Oncol. 2017, 12, 365-371. [CrossRef]

14. Kozak, K.; Kowalik, A.; Gos, A.; Wasag, B.; Lugowska, I.; Jurkowska, M.; Krawczynska, N.; Kosela-Paterczyk, H.; Switaj, T.; Teterycz, P.; et al. Cell-free DNA BRAF V600E measurements during BRAF inhibitor therapy of metastatic melanoma: Long-term analysis. Tumori. J. 2020, 106, 241-248. [CrossRef]

15. Braune, J.; Keller, L.; Schiller, F.; Graf, E.; Rafei-Shamsabadi, D.; Wehrle, J.; Follo, M.; Philipp, U.; Hussung, S.; Pfeifer, D.; et al. Circulating Tumor DNA Allows Early Treatment Monitoring in BRAF- and NRAS-Mutant Malignant Melanoma. JCO Precis. Oncol. 2020, 4, 20-31. [CrossRef]

16. Gray, E.S.; Rizos, H.; Reid, A.L.; Boyd, S.C.; Pereira, M.R.; Lo, J.; Tembe, V.; Freeman, J.; Lee, J.H.; Scolyer, R.A.; et al. Circulating tumor DNA to monitor treatment response and detect acquired resistance in patients with metastatic melanoma. Oncotarget 2015, 6, 42008-42018. [CrossRef]

17. Sanmamed, M.F.; Fernandez-Landazuri, S.; Rodriguez, C.; Zarate, R.; Lozano, M.D.; Zubiri, L.; Perez-Gracia, J.L.; Martin-Algarra, S.; Gonzalez, A. Quantitative cell-free circulating BRAFV600E mutation analysis by use of droplet digital PCR in the follow-up of patients with melanoma being treated with BRAF inhibitors. Clin. Chem. 2015, 61, 297-304. [CrossRef]

18. Lee, J.H.; Long, G.V.; Boyd, S.; Lo, S.; Menzies, A.M.; Tembe, V.; Guminski, A.; Jakrot, V.; Scolyer, R.A.; Mann, G.J.; et al. Circulating tumour DNA predicts response to anti-PD1 antibodies in metastatic melanoma. Ann. Oncol. 2017, 28, 1130-1136. [CrossRef] [PubMed]

19. Forthun, R.B.; Hovland, R.; Schuster, C.; Puntervoll, H.; Brodal, H.P.; Namlos, H.M.; Aasheim, L.B.; Meza-Zepeda, L.A.; Gjertsen, B.T.; Knappskog, S.; et al. ctDNA detected by ddPCR reveals changes in tumour load in metastatic malignant melanoma treated with bevacizumab. Sci. Rep. 2019, 9, 17471. [CrossRef]

20. McEvoy, A.C.; Warburton, L.; Al-Ogaili, Z.; Celliers, L.; Calapre, L.; Pereira, M.R.; Khattak, M.A.; Meniawy, T.M.; Millward, M.; Ziman, M.; et al. Correlation between circulating tumour DNA and metabolic tumour burden in metastatic melanoma patients. BMC Cancer 2018, 18, 726. [CrossRef] [PubMed]

21. Seremet, T.; Jansen, Y.; Planken, S.; Njimi, H.; Delaunoy, M.; El Housni, H.; Awada, G.; Schwarze, J.K.; Keyaerts, M.; Everaert, H.; et al. Undetectable circulating tumor DNA (ctDNA) levels correlate with favorable outcome in metastatic melanoma patients treated with anti-PD1 therapy. J. Transl. Med. 2019, 17, 303. [CrossRef] [PubMed] 
22. Rowe, S.P.; Luber, B.; Makell, M.; Brothers, P.; Santmyer, J.; Schollenberger, M.D.; Quinn, H.; Edelstein, D.L.; Jones, F.S.; Bleich, K.B.; et al. From validity to clinical utility: The influence of circulating tumor DNA on melanoma patient management in a real-world setting. Mol. Oncol. 2018, 12, 1661-1672. [CrossRef] [PubMed]

23. Santiago-Walker, A.; Gagnon, R.; Mazumdar, J.; Casey, M.; Long, G.V.; Schadendorf, D.; Flaherty, K.; Kefford, R.; Hauschild, A.; Hwu, P.; et al. Correlation of BRAF Mutation Status in Circulating-Free DNA and Tumor and Association with Clinical Outcome across Four BRAFi and MEKi Clinical Trials. Clin. Cancer Res. 2016, 22, 567-574. [CrossRef]

24. Chang, G.A.; Tadepalli, J.S.; Shao, Y.; Zhang, Y.; Weiss, S.; Robinson, E.; Spittle, C.; Furtado, M.; Shelton, D.N.; Karlin-Neumann, G.; et al. Sensitivity of plasma BRAFmutant and NRASmutant cell-free DNA assays to detect metastatic melanoma in patients with low RECIST scores and non-RECIST disease progression. Mol. Oncol. 2016, 10, 157-165. [CrossRef]

25. Ascierto, P.A.; Minor, D.; Ribas, A.; Lebbe, C.; O’Hagan, A.; Arya, N.; Guckert, M.; Schadendorf, D.; Kefford, R.F.; Grob, J.J.; et al. Phase II trial (BREAK-2) of the BRAF inhibitor dabrafenib (GSK2118436) in patients with metastatic melanoma. J. Clin. Oncol. 2013, 31, 3205-3211. [CrossRef]

26. Syeda, M.M.; Wiggins, J.M.; Corless, B.C.; Long, G.V.; Flaherty, K.T.; Schadendorf, D.; Nathan, P.D.; Robert, C.; Ribas, A.; Davies, M.A.; et al. Circulating tumour DNA in patients with advanced melanoma treated with dabrafenib or dabrafenib plus trametinib: A clinical validation study. Lancet Oncol. 2021, 22, 370-380. [CrossRef]

27. Gonzalez-Cao, M.; Mayo de Las Casas, C.; Jordana Ariza, N.; Manzano, J.L.; Molina-Vila, M.A.; Soriano, V.; Puertolas, T.; Balada, A.; Soria, A.; Majem, M.; et al. Early evolution of BRAFV600 status in the blood of melanoma patients correlates with clinical outcome and identifies patients refractory to therapy. Melanoma Res. 2018, 28, 195-203. [CrossRef]

28. Varaljai, R.; Wistuba-Hamprecht, K.; Seremet, T.; Diaz, J.M.S.; Nsengimana, J.; Sucker, A.; Griewank, K.; Placke, J.M.; Horn, P.A.; von Neuhoff, N.; et al. Application of Circulating Cell-Free Tumor DNA Profiles for Therapeutic Monitoring and Outcome Prediction in Genetically Heterogeneous Metastatic Melanoma. JCO Precis. Oncol. 2019, 3, PO.18.00229. [CrossRef]

29. Forschner, A.; Weißgraeber, S.; Hadaschik, D.; Schulze, M.; Kopp, M.; Kelkenberg, S.; Sinnberg, T.; Garbe, C.; Biskup, S.; Battke, F. Circulating Tumor DNA Correlates with Outcome in Metastatic Melanoma Treated by BRAF and MEK Inhibitors-Results of a Prospective Biomarker Study. OncoTargets Ther. 2020, 13, 5017-5032. [CrossRef] [PubMed]

30. Balch, C.M.; Gershenwald, J.E.; Soong, S.-J.; Thompson, J.F.; Atkins, M.B.; Byrd, D.R.; Buzaid, A.C.; Cochran, A.J.; Coit, D.G.; Ding, S.; et al. Final version of 2009 AJCC melanoma staging and classification. J. Clin. Oncol. 2009, 27, 6199-6206. [CrossRef]

31. Eisenhauer, E.A.; Therasse, P.; Bogaerts, J.; Schwartz, L.H.; Sargent, D.; Ford, R.; Dancey, J.; Arbuck, S.; Gwyther, S.; Mooney, M.; et al. New response evaluation criteria in solid tumours: Revised RECIST guideline (version 1.1). Eur. J. Cancer 2009, 45, 228-247. [CrossRef]

32. Van Wilpe, S.; Koornstra, R.; Den Brok, M.; De Groot, J.W.; Blank, C.; De Vries, J.; Gerritsen, W.; Mehra, N. Lactate dehydrogenase: A marker of diminished antitumor immunity. Oncoimmunology 2020, 9, 1731942. [CrossRef]

33. Gershenwald, J.E.; Scolyer, R.A.; Hess, K.R.; Sondak, V.K.; Long, G.V.; Ross, M.I.; Lazar, A.J.; Faries, M.B.; Kirkwood, J.M.; McArthur, G.A.; et al. Melanoma staging: Evidence-based changes in the American Joint Committee on Cancer eighth edition cancer staging manual. CA Cancer J. Clin. 2017, 67, 472-492. [CrossRef] [PubMed]

34. Nosrati, A.; Tsai, K.K.; Goldinger, S.M.; Tumeh, P.; Grimes, B.; Loo, K.; Algazi, A.P.; Nguyen-Kim, T.D.L.; Levesque, M.; Dummer, R.; et al. Evaluation of clinicopathological factors in PD-1 response: Derivation and validation of a prediction scale for response to PD-1 monotherapy. Br. J. Cancer 2017, 116, 1141-1147. [CrossRef]

35. Bucheit, A.D.; Syklawer, E.; Jakob, J.A.; Bassett, R.L., Jr.; Curry, J.L.; Gershenwald, J.E.; Kim, K.B.; Hwu, P.; Lazar, A.J.; Davies, M.A. Clinical characteristics and outcomes with specific BRAF and NRAS mutations in patients with metastatic melanoma. Cancer 2013, 119, 3821-3829. [CrossRef] [PubMed]

36. Calbet-Llopart, N.; Potrony, M.; Tell-Marti, G.; Carrera, C.; Barreiro, A.; Aguilera, P.; Podlipnik, S.; Puig, S.; Malvehy, J.; PuigButille, J.A. Detection of cell-free circulating BRAF(V) $(600 \mathrm{E})$ by droplet digital polymerase chain reaction in patients with and without melanoma under dermatological surveillance. Br. J. Dermatol. 2020, 182, 382-389. [CrossRef]

37. Wong, S.Q.; Raleigh, J.M.; Callahan, J.; Vergara, I.A.; Ftouni, S.; Hatzimihalis, A.; Colebatch, A.J.; Li, J.; Semple, T.; Doig, K.; et al. Circulating Tumor DNA Analysis and Functional Imaging Provide Complementary Approaches for Comprehensive Disease Monitoring in Metastatic Melanoma. JCO Precis. Oncol. 2017, 1, 1-14. [CrossRef] 